\title{
A New Analysis Method for Complex Network Based on Dynamics of Spin Diffusion
}

\author{
Makoto Uchida $^{1}$ and Susumu Shirayama ${ }^{2}$ \\ ${ }^{1}$ Graduate School of Engineering, the University of Tokyo, \\ 5-1-5 Kashiwanoha, Kashiwa city, Chiba, 277-8568, Japan \\ uchida@race.u-tokyo.ac.jp \\ 2 Research into Artifacts, Center for Engineering, the University of Tokyo, \\ 5-1-5 Kashiwanoha, Kashiwa city, Chiba, 277-8568, Japan \\ sirayama@race.u-tokyo.ac.jp
}

\begin{abstract}
We propose a new analysis method for a complex network based on a simple spin diffusion model. The model is constructed by a local interaction between vertices, as is in the spin dynamics. Several numerical experiments on network models are performed systematically under various initial conditions. The results strongly depend on the network structures, also on the initial conditions, while most conventional measures of the networks are almost same. It may be shown that the difference of dynamics induced by such interaction reveals a new characteristic feature of the network structure.
\end{abstract}

\section{Introduction}

Complex network analysis methods have been utilized to inquire complicated structures and functions. Early works had shown that many real-world networks in various fields have the common structural properties, typically such as 'small-world', 'scale-free' or community structure [1, 2]. Recently, various methods or new measures for analyzing a unique structure of networks are being proposed. Other works have paid attention to phenomena on networks; percolation, epidemic spreading, traffic on networks, etc [2]. A fundamental problem is to understand how the topological structure affects the dynamics that it supports [3].

In this paper, we propose a simple model of diffusion on networks, based on a local interaction between vertices, then apply this model on several models of network numerically. The resulting phenomena are very different depending on both initial conditions and the structure of networks itself. The difference of the diffusion dynamics by our method may reveal a new characteristic of the network structure.

\section{Proposed Method}

\subsection{Diffusion Model}

We consider a simple diffusion process on networks. First, a variable $\sigma_{i}(t)$ which takes +1 or -1 is defined. This variable represents a certain state of an vertex $v_{i}$ at a time step $t$. Then, we set $\sigma_{i}(0)$ to all vertices as an initial condition in some manners. In 
the consequent section, we explain the details of the initial condition. The state at the subsequent step $t+1$ is determined by the present state of vertices to which an edge from $v_{i}$ exists. There is an interaction only among neighboring vertices. $\sigma_{i}(t+1)$ is determined by the following rule:

$$
\begin{aligned}
& \sigma_{i}(t+1)=\operatorname{sgn}\left\{\sum_{j}^{N} a_{i j} \sigma_{j}(t)\right\} \\
& \sigma_{i}(t+1)=\sigma_{i}(t), \text { if } \sum_{j}^{N} a_{i j} \sigma_{j}(t)=0
\end{aligned}
$$

where $a_{i j}=a_{j i}$ is the $i-j(j-i)$ element of an adjacency matrix, which takes the value of 1 if there is an edge between vertices $v_{i}$ and $v_{j}$, otherwise 0 . The states of vertices are updated synchronously at each step.

\subsection{Initial Conditions}

We give the initial ratio $(r)$ of the vertices with +1 . In this paper, two types of initial conditions are applied for the initial state of the vertices. One is the random distribution, where the $r N$ vertices out of the whole $N$ vertices are randomly chosen and given +1 . The other is selecting the vertices to be +1 corresponding to a local property of a network. Four centrality measures; degree centrality, closeness centrality, betweenness centrality [1,4] and clustering coefficient [5] are used in our numerical studies. The $r N$ vertices with the largest centrality are given +1 as an initial state, while the rest $(1-r) N$ vertices are given -1 .

\section{Numerical Studies Using Network Models}

Numerical studies are performed using four theoretical, computer-generated network models; random graph, Barabási-Albert model [6], Klemm-Eguílz model $(\mu=0.1)$ [7] and CNN (Connecting Nearest Neighbor) model [8], with the vertices $N=36000$ and the average degree $\langle k\rangle=10$. It is known that they all have 'small-world' property and the three network models except random graph have power-law degree distribution.

The numerical results are as follows. Typical results of the time-progress transition of the ratio of +1 are shown in Fig. 1. Fig. 2] shows the convergence ratio of the state +1 as a function of the initial ratio $r$, on each initial conditions. Some cases have an oscillation in convergence. We consider the average of the ratio of the last two step as a convergence ratio if it oscillates.

On random graph and BA model network, the +1 state has been extinguished or throughly spread in the whole network, while on KE model and CNN model two states are co-existing in convergence. On KE model with a certain initial conditions, a strong, damped oscillation mode is observed, while there is no or little oscillation on other networks. A dynamics and a convergence of the states are also affected by the centrality of the vertices which have initial states. It determines whether the state +1 will spread or deflate. 


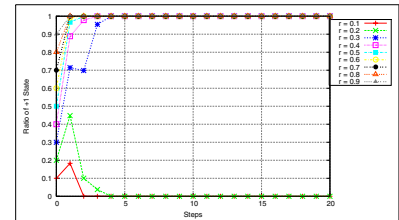

BA Model (degree)

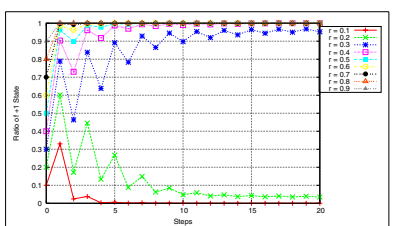

KE Model (degree)

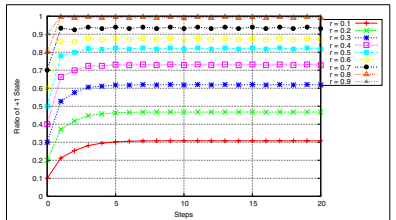

CNN Model (degree)

Fig. 1. Typical results of the transition of the ratio of the state +1 on network models. Each caption describes a network model and an initial condition.

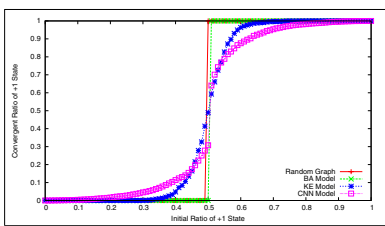

Random

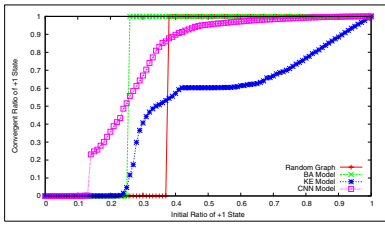

Betweenness

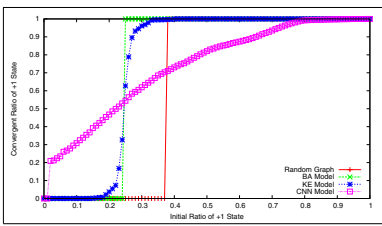

Degree

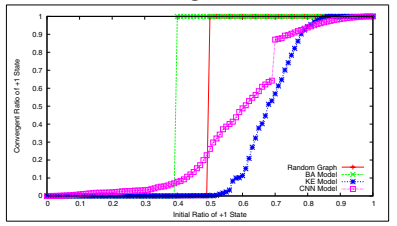

Clustering

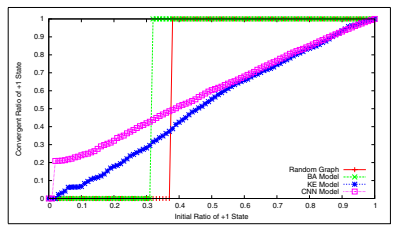

Closeness

Fig. 2. Convergence ratio of the state +1 on network models by each initial conditions. Horizontal and vertical axes indicate the initial and the convergence ratio. Random graph, BA model, KE model and CNN model are represented as red, green, blue and purple dot, respectively.

\section{Results}

As shown in the previous section, the dynamics caused by our model strongly depends on the initial conditions and the network structures. It is found that patterns from the relations among initial condition, $r$ and the convergence ratio are classified into several classes (Fig. 3).
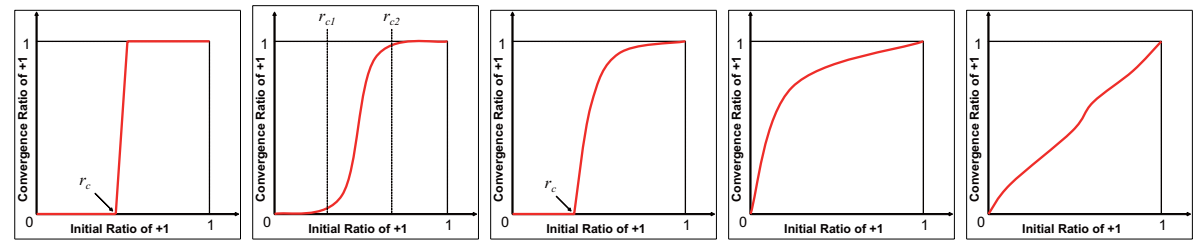

Fig. 3. Several classes are derived from the numerical results. Typical patterns are shown above. 
On random graph and BA model, it is like a step function with all initial conditions, while convex curve or sigmoid-function-like curve are observed on KE model and CNN model with several initial conditions. In some cases, such as CNN model with betweenness-order initial distribution, they have non-trivial critical values to change the state. The absorbing state (all vertices take +1 or -1 ) in random graph and BA model might be related to low clustering coefficient. It is quite difficult to explain the oscillation or the co-existence of two states in fully developed stage from the conventional structural measures of network.

\section{Conclusions}

A new method to analyze a network structure from the patterns derived from the simple two-state diffusion process is proposed. Numerical studies are carried out using various network models. The phenomena appeared on the networks strongly depend on the initial conditions and the network structures. We classify the numerical results into several classes. The pattern in the classes may be connected with new structural properties.

It is considered that the diffusion dynamics by our method reveals some novel structural characteristics, and it gives us a clue for exploring the relations between a structure and a function of network.

\section{References}

1. Wasserman, S., Faust, K.: Social Network Analysis: Methods and Applications. Cambridge University Press (1994)

2. Newman, M.E.J.: The structure and function of complex networks. SIAM Review 45 (2003) $167-256$

3. Tadíc, B., Thurner, S.: Information super-diffusion on structured networks. Physica A (332) (2004) $566-584$

4. Brandes, U.: A faster algorithm for betweenness centrality. Journal of Mathematical Sociology 25(2) (2001) $163-177$

5. Watts, D.J., Strogatz, S.H.: Collective dynamics of 'small-world' networks. Nature 393 (1998) $440-442$

6. Barabási, A.L., Albert, R.: Emergence of scaling in random networks. Science 286 (1999) $509-512$

7. Klemm, K., Eguíluz, V.M.: Highly clustered scale-free networks. Physical Review E 65(036123) (2002)

8. Vázquez, A.: Growing network with local rules: Preferential attachment, clustering hierarchy, and degree correlations. Physical Review E 67(056104) (2003) 